\section{Prospectiva estratégica del cluster de muebles de Villa El Salvador}

\author{
Recepción: Mayo de $2006 /$ Aceptación: Junio de 2006
}

(1) Jorge Inche Mitma
(2) Alfonso Chung Pinzás
(3) Julio Salas Bacalla

\section{RESUMEN}

El presente artículo aborda el siguiente problema ¿Cómo formular un escenario al año 2015 que sea realista y a la vez sea la base para una visión factible de cumplirse para el cluster de muebles de Villa EISalvador?; para la obtención de los resultados se aplicaron encuestas DELPHIy MINIDEPLPHI así como el método de la Prospectiva Estratégica, la cual incluye los métodos MICMAC, MACTORy SMIC, todos los datos fueron procesados mediante software libre del instituto LIPSOR deFrancia. Los principales resultados obtenidos fueron: las variables del eje estratégico, rol de actores, el escenario apuestaal 2015 y una propuesta de visión a dicho año.

Palabras Clave: Prospectiva, cluster, actores, escenarios, variables.

Strategic Prospective of Villa El SALVAdOR FURNITURE CLUSTER ABSTRACT

This article focuses the next problem: How to formulate a realistic scenery to 2015 and at the same time be the base of a vision that can be carry out to the furniture cluster of Villa El Salvador?; in order to have results, we applied DELPHI and MINI DELPHI surveys as well the prospective strategic method, it involves the MIC MAC, MACTOR and SMIC methods; all the data was processes by shareware software of LIPSOR institute of France. The principal results were: the variables of the strategic axis, the actors role, the bets scenery to 2015 and a vision proposed to that year.

Key words: Prospective, cluster, actors, scenarios, variable.

\section{INTRODUCCIÓN}

El estudio de la Prospectiva Estratégica en el Cluster de Muebles de Villa el Salvador es importante porque permite obtener un escenario futuro que no sea fruto del simple proceso de imaginación o de prospección mental de los actores, sino un escenario basado en la cuantificación de las opiniones cualitativas y en las probabilidades. El principal autor de este método es el Dr. Michael Godet del Instituto LIPSOR (Laboratoire d'investigation en prospective strategie et organisation) y ya ha sido usado en diversos países como Francia, Venezuela, Colombia entre otros.

El objetivo del estudio que da como origen el presente artículo es formular un escenario realista al 2015 para el Cluster de Muebles de Villa El Salvador, se justifica el estudio puesto que la mayoría de planes estratégicos parten de una visión errada y muchas veces imposible de cumplir, debido que sólo obedecen a anhelos y procesos mentales sin un soporte estadístico adecuado, con lo cual todo su plan estratégico está errado y con ello sus planes y programas.

Por otro lado es importante para toda organización el adelantarse al futuro a fin de obtener uno o varios escenarios "futuribles" eligiendo el escenario apuesta, pero con una sólida base estadística, con lo cual sea más factible de llevarse a la práctica. Cabe mencionar que el sustento base de la prospectiva estratégica es que el futuro no está escrito, ni es uno sólo sino son varios escenarios "futuribles" con lo cual la organización debe escoger a donde desea llegar o construir su propio escenario dentro de los escenarios mas probables.Las limitaciones del estudio están en la disponibilidad del llenado de encuestas de los principales actores.

Para la realización del estudio se dividió en las siguientes fases:

Fase 0: Conformación de los equipos de trabajo

Fase I: Análisis Estructural y aplicación del método MIC MAC

Fase II: Análisis del Rol de Actores mediante el método MACTOR

Fase III: Escenarios Futuros y Escenario Apuesta método SMIC

\section{FASE I : ANÁLISIS ESTRUCTURAL Y EL MÉTODO MIC MAC}

El Análisis Estructural es el punto de partida de todo estudio prospectivo estratégico y permite obtener las variables del eje estratégico, es decir

(1) Magíster en Ciencias. Profesor del Departamento de Diseño y Tecnología Industrial, UNMSM E-mail: jlinche@hotmail.com

(2) Magíster en Ingeniería Industrial. Jefe de la Oficina de Planificación, Presupuesto y Racionalización de la Facultad de Ingeniería Industrial, UNMSM.

E-mail: ramon_chung@yahoo.es

(3) Ingeniero Industrial. Profesor del Departamento de Producción y Gestión Industrial, UNMSM. E-mail: jasalasb@hotmail.com 
aquellas que son las más inestables del sistema. Para el caso en estudio se aplicaron encuestas DELPHI a empresarios de muebles, funcionarios de CITEMADERAS y a dirigentes de gremios de fabricantes, a fin de obtener las variables más importantes del sistema, llegándose a identificar 35 variables las cuales se presentan a continuación:

Variable 01: Costo de Materia Prima

Variable 02: Flete de Transporte

Variable 03: Demanda de Muebles

Variable 04: Renovación de tecnología

Variable 05: Políticas económicas

Variable 06: Certificaciones para al exportación

Variable 07: Captación de mercados internacionales

Variable 08: Nivel de capacitación

Variable 09: Calidad de los productos

Variable 10: Gestión administrativa

Variable 11: Formalización de empresas

Variable 12: Seguridad de la empresa

Variable 13: Visión del empresario con respecto al mercado

Variable 14: Integración de las empresas de diseño con los distribuidores

Variable 15: Acceso a la información de mercado

Variable 16: Precio de insumos para el acabado

Variable 17: Capital de reinversión

Variable 18: Tipo de madera

Variable 19: Satisfacción del cliente

Variable 20: Financiamiento de proyectos

Variable 21: Donaciones de cooperación internacional

Variable 22: Procesos de manufactura

Variable 23: Publicidad

Variable 24: Sistema de seguros contra imprevistos

Variable 25: Sistema de información gerencial

Variable 26: Idioma

Variable 27: Diversificación de productos

Variable 28: Volumen de ventas

Variable 29: Utilidad

Variable 30: Flexibilidad al cambio

Variable 31: Ubicación geográfica

Variable 32: Condiciones de trabajo

Variable 33: Edad de los trabajadores

Variable 34: Nivel de experiencia de los trabajadores

Variable 35: Investigación y desarrollo de nuevas tecnologías y productos

Luego se aplicó la matriz de impactos directos MID la cual consiste en una matriz de doble entrada en donde se cruzan las variables para hallar su influencia sobre la otra bajo el siguiente sistema de calificación:

$0=$ no influye

1 = influencia débil

2 = influencia media

3 = influencia fuerte

$\mathrm{P}=$ influencia potencial
Sin embargo, la MID sólo toma en consideración las influencias directas quedando fuera las indirectas, por lo tanto aplicando el método MIC MAC, el cual consiste multiplicar la matriz por si misma hasta encontrar un cierto equilibrio, se pudo encontrar la matriz de impactos indirectos o MII cuyos resultados son más completos (ver cuadros 1 y 2 respectivamente).

Es importante tener en cuenta que tanto en la MID como la MII la suma de las filas proporciona el valor de dependencia de una variable y la sumatoria de las columnas su valor de influencia, con esto se obtiene un par ordenado $\mathrm{x}, \mathrm{y}$ el cual pude ser graficado en un plano cartesiano tal y como se presenta en la figura 1. En dicha figura se puede apreciar que según su ubicación, las variables se clasifican en:

Variables Determinantes: Aquellas que tienen una fuerte motricidad y son poco o nada dependientes de alguna otra variable dentro del sistema.

Variables Autónomas: Aquellas que tienen baja motricidad y baja influencia dentro del sistema.

Variables Reguladoras y/o Palancas: Son consideradas como "llaves de paso" debido a su nivel de equilibrio en cuanto a motricidad y dependencia y son aquellas que determinan, en condiciones normales, el funcionamiento y desarrollo del sistema.

Variables Resultado: Son aquellas con una fuerte dependencia y una baja influencia, es decir aquellas que resultan de los cambios de otras variables.

Variables Claves: Las variables claves son las más inestables del sistema y por lo tanto las mas peligrosas, ya que son aquellas que tiene una fuerte influencia pero también una fuerte dependencia.

Variables de Entorno: Aquellas propias del sistema o que también son parte reguladora, tienen poca dependencia y una influencia media

El siguiente paso consiste en encontrar las variables estratégicas, es decir aquellas que tienen los mayores valores estratégicos, entendiéndose como valor estratégico la sumatoria de su influencia más su dependencia, los resultados se presentan a continuación:

1. Investigación y desarrollo de nuevas tecnologías y productos

2. Procesos de manufactura

3. Renovación de tecnología

4. Visión del empresario con respecto al mercado

5. Donaciones de cooperación internacional

6. Flexibilidad al cambio

7. Financiamiento de proyectos 
Cuadro 1. Matriz MID

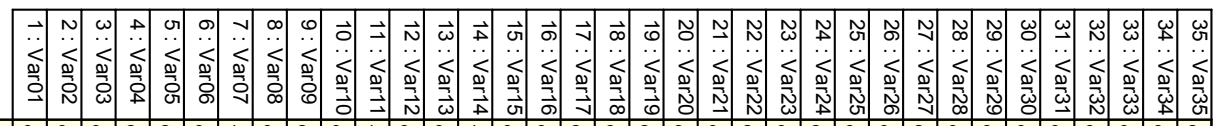

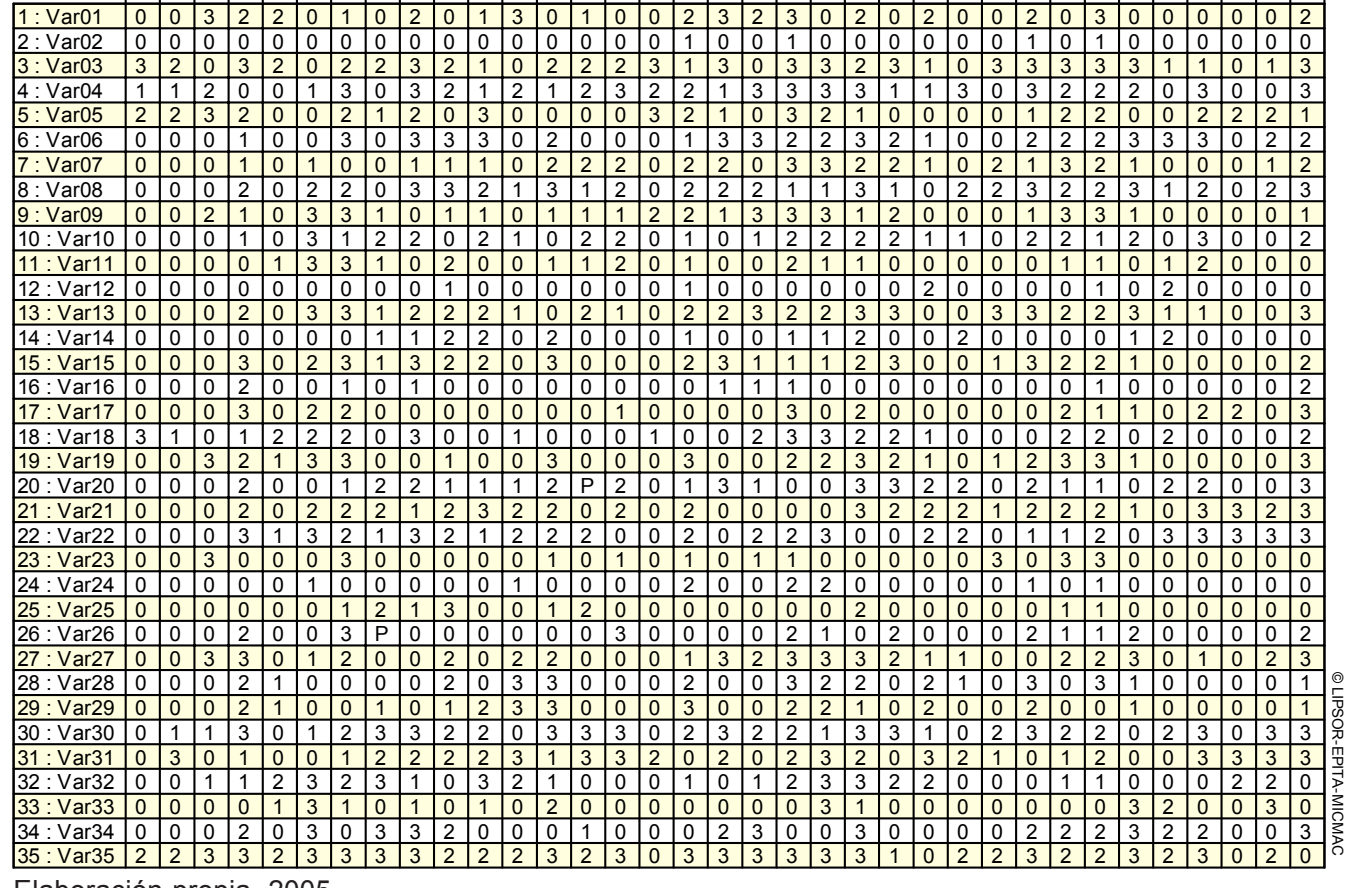

Elaboración propia, 2005

Cuadro 2. Matriz MII

\begin{tabular}{|c|c|c|c|c|c|c|c|c|c|c|c|c|c|c|c|c|c|c|}
\hline & 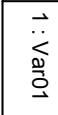 & 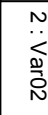 & 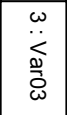 & 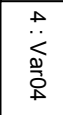 & 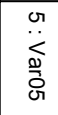 & 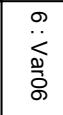 & 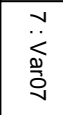 & $\begin{array}{l}\infty \\
\ddot{\infty} \\
\stackrel{\infty}{\grave{c}} \\
\infty\end{array}$ & 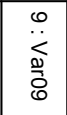 & 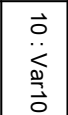 & 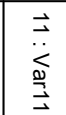 & 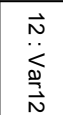 & 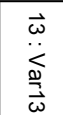 & 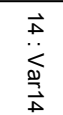 & 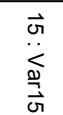 & 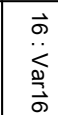 & 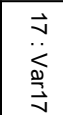 & 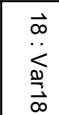 \\
\hline $1: \operatorname{Var} 01$ & 443 & 473 & 1003 & 2271 & 657 & 1895 & 2371 & 1367 & 2011 & 1835 & 1646 & 1482 & 2064 & 1142 & 1501 & 488 & 2075 & 1620 \\
\hline $2: \operatorname{Var} 02$ & 59 & 55 & 103 & 220 & 74 & 214 & 249 & 145 & 226 & 191 & 157 & 152 & 207 & 133 & 162 & 46 & 212 & 159 \\
\hline $3: \operatorname{Var} 03$ & 814 & 791 & 1848 & \begin{tabular}{|l|}
4142 \\
\end{tabular} & \begin{tabular}{|l|}
1219 \\
\end{tabular} & 3676 & \begin{tabular}{|l|}
4465 \\
\end{tabular} & 2490 & \begin{tabular}{|l|}
3648 \\
\end{tabular} & 3469 & 2915 & 2568 & 3920 & 2088 & 2716 & 829 & 3829 & 2912 \\
\hline $4: \operatorname{Var} 04$ & 694 & 699 & 1558 & 3614 & 1029 & 3282 & 3960 & 2167 & 3292 & 3096 & 2659 & 2316 & 3413 & 1892 & 2452 & 728 & 3371 & 2520 \\
\hline $5: \operatorname{Var} 05$ & 474 & 488 & 1096 & 2437 & 696 & 2156 & 2617 & 1479 & 2229 & 2042 & 1806 & 1550 & 2287 & 1238 & 1581 & 491 & 2271 & 1743 \\
\hline $6: \operatorname{Var} 06$ & 609 & 628 & 1435 & 3430 & 987 & 3083 & 3710 & 2101 & 3111 & 2977 & 2610 & 2164 & 3342 & 1680 & 2229 & 639 & 3221 & 2409 \\
\hline $7: \operatorname{Var} 07$ & 413 & 463 & 1093 & 2368 & 676 & 2184 & 2628 & 1452 & \begin{tabular}{|l|}
2128 \\
\end{tabular} & 2043 & 1744 & 1535 & 2276 & 1282 & 1616 & 454 & 2228 & 1693 \\
\hline 8: Var08 & 662 & 702 & 1620 & 3694 & 1054 & 3335 & 4019 & 2273 & 3347 & 3181 & 2765 & 2324 & 3598 & 1910 & 2476 & 704 & 3456 & 2616 \\
\hline 9: Var09 & 500 & 521 & 1194 & 2688 & 773 & 2431 & 2909 & 1634 & 2357 & 2212 & 1899 & 1750 & 2463 & 1419 & 1815 & 496 & 2472 & 1847 \\
\hline $10: \operatorname{Var} 10$ & 462 & 488 & 1055 & 2581 & 736 & 2451 & 2824 & 1613 & 2340 & 2223 & 1894 & 1620 & 2414 & 1407 & 1739 & 506 & 2372 & 1778 \\
\hline $11:$ Var11 & 264 & 280 & 694 & 1540 & 463 & 1579 & 1781 & 1001 & 1402 & 1357 & 1136 & 1013 & 1485 & 832 & 1071 & 272 & 1457 & 1044 \\
\hline $12: \operatorname{Var} 12$ & 46 & 42 & 109 & 320 & 94 & 329 & 345 & 231 & 305 & 294 & 266 & 207 & 325 & 137 & 207 & 35 & 309 & 224 \\
\hline $13:$ Var13 & 658 & 696 & 1512 & 3599 & 1032 & 3224 & 3849 & 2251 & 3224 & 3048 & 2632 & 2268 & 3413 & 1879 & 2400 & \begin{tabular}{|l|}
691 \\
\end{tabular} & 3313 & 2544 \\
\hline $14:$ Var14 & 171 & 195 & 482 & 1129 & 340 & 1160 & 1289 & 766 & 1091 & 1049 & 941 & 729 & 1181 & 600 & 765 & 180 & 1097 & 806 \\
\hline $15: \operatorname{Var} 15$ & 532 & 553 & 1284 & 2996 & 857 & 2532 & 3142 & 1816 & 2582 & 2470 & 2152 & 1851 & 2873 & 1475 & 1956 & 538 & 2751 & 2089 \\
\hline $16: \operatorname{Var} 16$ & 154 & 149 & 358 & 836 & 232 & 673 & 869 & 476 & 701 & 675 & 577 & 490 & 757 & 393 & 499 & 165 & 744 & 579 \\
\hline $17: \operatorname{Var} 17$ & 318 & 349 & 789 & 1787 & 495 & 1716 & 1987 & 1153 & 1622 & 1554 & 1325 & 1106 & 1632 & 957 & 1160 & 376 & 1636 & 1251 \\
\hline $18: \operatorname{Var} 18$ & 479 & 448 & 951 & 2329 & 715 & 2107 & 2446 & 1411 & 2008 & 1916 & 1616 & 1467 & 2144 & 1148 & 1486 & 478 & 2126 & 1570 \\
\hline 19: Var19 & 554 & 583 & 1348 & 3023 & 872 & 2717 & 3252 & 1889 & 2672 & 2493 & 2161 & 1924 & 2805 & 1552 & 2043 & 571 & 2753 & 2108 \\
\hline $20:$ Var20 & 458 & 469 & 1076 & 2605 & 732 & 2269 & 2757 & 1580 & 2294 & 2211 & 1941 & 1598 & 2493 & 1295 & 1705 & 511 & 2424 & 1816 \\
\hline $21:$ Var21 & 503 & 575 & 1253 & 3052 & 813 & 2776 & 3294 & 1926 & 2779 & 2656 & 2336 & 1869 & 2917 & 1605 & 2052 & 585 & 2800 & 2179 \\
\hline 22: Var22 & 560 & 616 & 1363 & 3396 & 973 & 3181 & 3665 & 2167 & 3099 & 2960 & 2547 & 2088 & 3264 & 1788 & 2213 & 597 & 3115 & 2354 \\
\hline $23:$ Var23 & 228 & 227 & 589 & 1274 & 293 & 1007 & 1332 & 697 & 1067 & 983 & 853 & 824 & 1114 & 600 & 876 & 205 & 1153 & 896 \\
\hline $24:$ Var24 & 115 & 126 & 261 & 548 & 194 & 592 & 639 & 375 & 548 & 487 & 406 & 392 & 540 & 343 & 394 & 100 & 534 & 388 \\
\hline $25:$ Var25 & 114 & 154 & 387 & 887 & 225 & 853 & 1013 & 597 & 786 & 820 & 693 & 555 & 870 & 473 & 615 & 141 & 824 & 624 \\
\hline $26:$ Var26 & 329 & 284 & 676 & 1587 & 464 & 1419 & 1731 & 919 & 1381 & 1301 & 1056 & 968 & 1433 & 799 & 1024 & 305 & 1420 & 1062 \\
\hline $27:$ Var27 & 583 & 646 & 1537 & 3319 & 958 & 2977 & 3612 & 2013 & 2928 & 2789 & 2436 & 2143 & 3123 & 1691 & 2168 & 660 & 3081 & 2322 \\
\hline $28:$ Var28 & 350 & 375 & 850 & 1870 & 569 & 1791 & 2089 & 1209 & 1720 & 1631 & 1382 & 1201 & 1808 & 1008 & 1283 & 348 & 1746 & 1314 \\
\hline $29:$ Var29 & 299 & 325 & 731 & 1662 & 515 & 1547 & 1827 & 1069 & 1535 & 1427 & 1244 & 1054 & 1638 & 880 & 1113 & 295 & 1527 & 1189 \\
\hline $30:$ Var30 & 754 & 796 & 1839 & 4226 & \begin{tabular}{|l|}
1199 \\
\end{tabular} & 3743 & 4566 & 2575 & 3765 & 3602 & 3103 & 2626 & 4064 & 2184 & 2808 & 800 & 3921 & 2974 \\
\hline $31:$ Var31 & 441 & 543 & 1249 & 2869 & 752 & 2695 & 3211 & 1775 & 2632 & 2569 & 2219 & 1804 & 2750 & 1557 & 1935 & 559 & 2676 & 2063 \\
\hline $32:$ Var32 & 388 & 467 & 987 & 2374 & 690 & 2232 & 2545 & 1537 & 2115 & 2009 & 1776 & 1530 & 2234 & 1277 & 1590 & 436 & 2148 & 1662 \\
\hline $33:$ Var33 & 277 & 307 & 716 & 1586 & 518 & 1650 & 1793 & 1052 & 1481 & 1385 & 1199 & 1039 & 1579 & 857 & 1072 & 282 & 1486 & 1085 \\
\hline $34:$ Var34 & 526 & 560 & 1260 & 2936 & 838 & 2718 & 3169 & 1883 & 2636 & 2522 & 2176 & 1815 & 2747 & 1533 & 1922 & 585 & 2710 & 2054 \\
\hline $35:$ Var35 & 942 & 969 & 2258 & 5036 & \begin{tabular}{|l|}
1493 \\
\end{tabular} & 4602 & 5457 & 3096 & 4507 & 4229 & 3653 & 3229 & \begin{tabular}{|l}
4811 \\
\end{tabular} & 2613 & 3364 & 959 & 4698 & 3511 \\
\hline
\end{tabular}




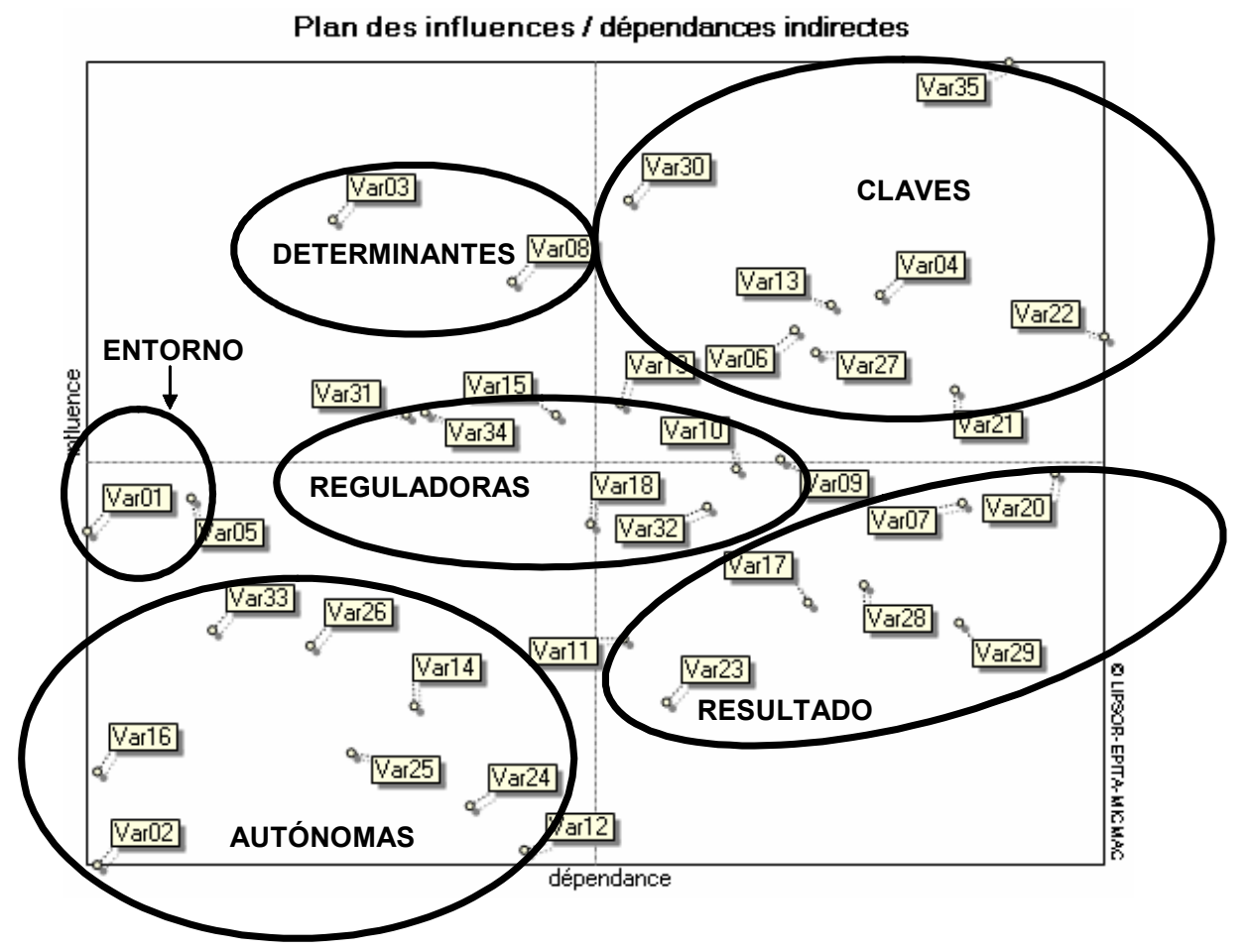

Figura 1. Clasificación de variables en el plano

8. Certificaciones para la exportación

9. Diversificación de productos

10.Captación de mercados internacionales

11.Nivel de capacitación

12.Calidad de los productos

13.Demanda de muebles

14. Gestión administrativa

15.Satisfacción del cliente

FASE II: JUEGO DE ACTORES - MÉTODO MACTOR

La segunda parte de esta investigación debe conducir a proporcionar información importante sobre los principales actores involucrados en el sistema estudiado y dentro de las 15 variables del eje estratégico. Aplicando una encuesta a los expertos, se obtiene la siguiente lista de actores:
1. CITEMADERAS
2. Universidades
3. Institutos Superiores
4. CONCYTEC
5. Bancos
6. Certificadoras
7. PROMPYME
8. Operarios
9. Empresarios
10. ONGs y Fundaciones

11. Gobierno Central
12. PROMPEX
13. Proveedores
14. APEMIVES

Luego se aplica la matriz de ActoresxActores (MAA) la cual relaciona a los actores según la siguiente calificación:

4: el actor $A$ puede cuestionar la existencia del actor $A$ 3: el actor $A$ puede cuestionar las misiones del actor $A$ 2: el actor $A$ puede cuestionar los proyectos del actor $A$ 1: el actor $A$ puede cuestionar, de manera limitada (durante algún tiempo o en algún caso concreto) la operativa del actor $A$.

0 : el actor $A_{i}$ no tiene ninguna influencia sobre el actor $A_{i}$

Sin embargo esta matriz sólo implica las relaciones directas, mas no las indirectas, por lo tanto es necesario aplicar la Matriz de Impacto Directo e Indirecto (MIDI) que es elevar al cuadro sucesivamente la MAA hasta lograr un cierto equilibrio. Los resultados de la MAA y de la MIDI se pueden apreciar en los cuadros 3 y 4 respectivamente.

Así la suma de las filas proporciona el grado total de influencia de un actor sobre el sistema $\left(I_{i}\right)$ y la suma de las columnas su grado de dependencia $\left(D_{i}\right)$; en la figura 2 se puede apreciar la clasificación de los actores de acuerdo a su posición en el plano. 
Cuadro 3. Matriz MAA

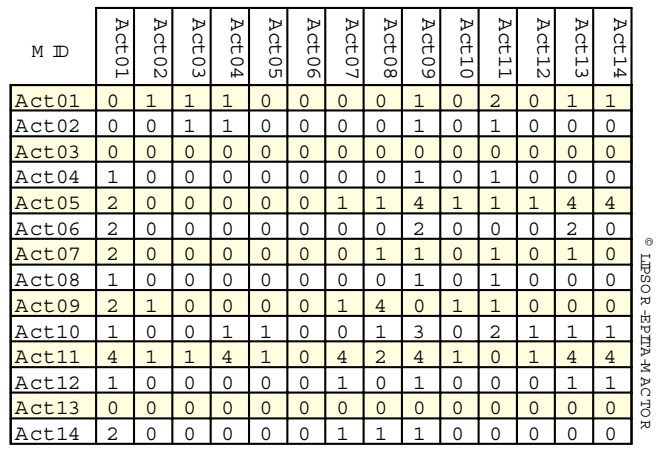

Elaboración propia, 2005

FASE III : ESCENARIOS FUTUROS Y ESCENARIO APUESTA - MÉTODO SMIC

El objetivo del presente estudio es proponer un escenario apuesta el cual sea matemáticamente probable de realizarse; así dentro de las 15 variables del eje estratégico, las 5 primeras serán la base para formular el escenario apuesta.

Para llevar a cabo este propósito, cada variable da origen a una hipótesis y de la combinación de hipótesis nacerán los posibles escenarios y de su evaluación probabilística se escogerá el escenario más probable.
Cuadro 4. Matriz MIDI

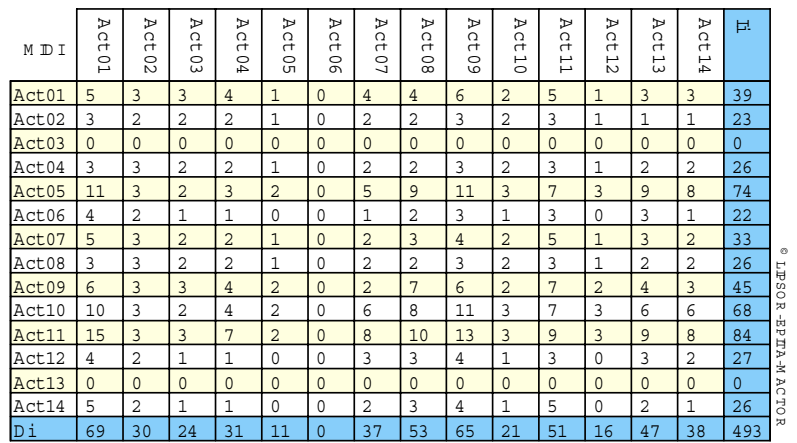

Elaboración propia, 2005

El cuadro 5 presenta las variables y las hipótesis que de ellas se formulan, recordando que un escenario es la combinación de las hipótesis que se presentan en dicho cuadro. Por otro lado el método SMIC está conformado por un juego de probabilidades que incluye : la probabilidad de realización de cada hipótesis por separado, la probabilidad de realización de una hipótesis si se realiza otra (si realización), la probabilidad de realización de una hipótesis si no se realiza otra (no realización) y de las probabilidades de realización de las $2 n$ posibles combinaciones (escenarios). Así para el caso de estudio se tienen 5 variables por lo tanto $25=32$ posibles escenarios al

Plan des influences et dépendances entre acteurs

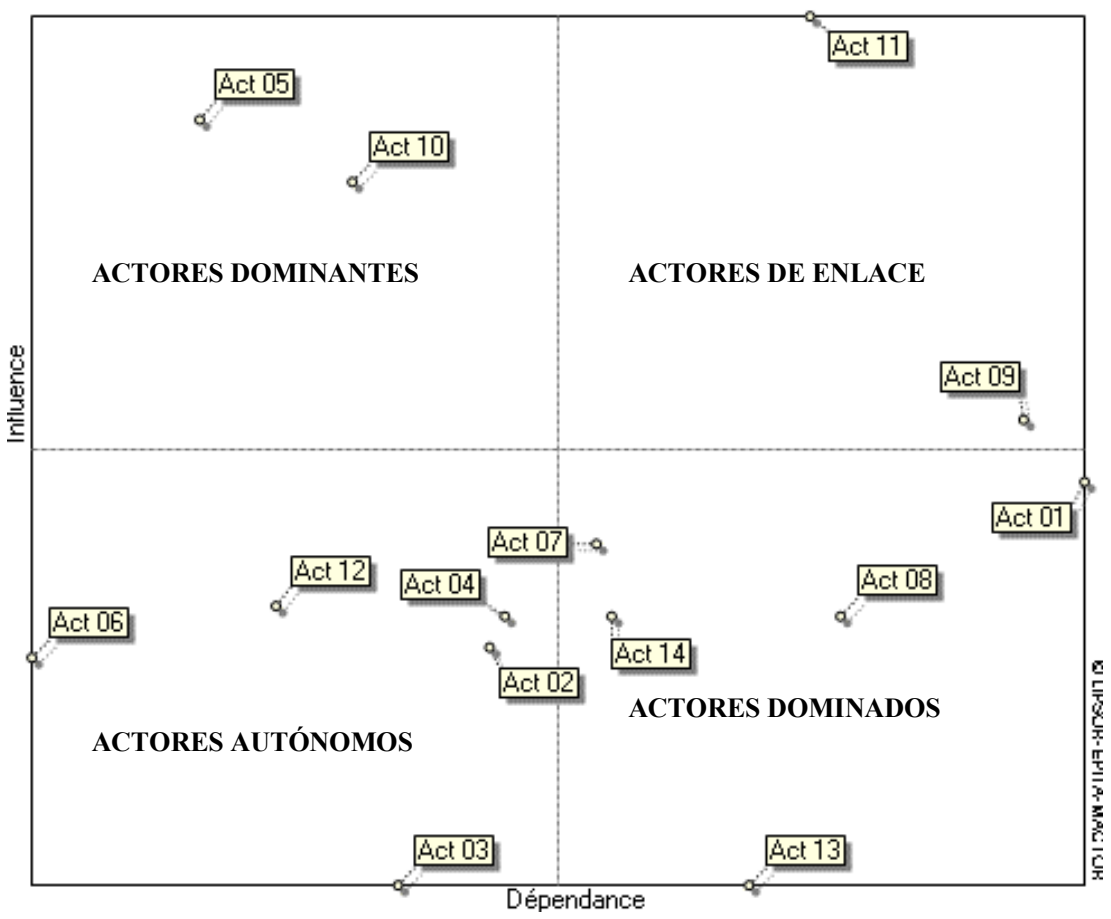

Figura 2. Plano de influencia y dependencia de actores 
Cuadro 5. Hipótesis formuladas

\begin{tabular}{|c|c|c|c|}
\hline No. & Inicial & Nombre & Hipótesis \\
\hline 1 & Var1 & $\begin{array}{l}\text { Investigación y Desarrollo } \\
\text { de Nuevas Tecnologías y } \\
\text { Productos }\end{array}$ & $\begin{array}{l}\text { En el año } 2015 \text { el cluster de muebles de } \\
\text { Villa El Salvador será un emporio } \\
\text { empresarial en donde la investigación y } \\
\text { desarrollo de nuevas tecnologías habrán } \\
\text { alcanzado avances bastante notorios y la } \\
\text { frecuencia de realización es constante, } \\
\text { contando con profesionales idóneos para el } \\
\text { caso y con convenios con CONCYTEC, } \\
\text { institutos y universidades nacionales e } \\
\text { internacionales }\end{array}$ \\
\hline 2 & Var2 & Procesos de manufactura & $\begin{array}{l}\text { En el año } 2015 \text { los procesos de manufactura } \\
\text { en los clusters de muebles de Villa El } \\
\text { Salvador cumplirán con los estándares } \\
\text { internacionales tanto de calidad como de } \\
\text { buenas prácticas, asimismo la producción } \\
\text { será en serie, por otro lado la merma se } \\
\text { verá reducida por exigentes programas de } \\
\text { productividad otorgando mayor valor } \\
\text { agregado al producto. }\end{array}$ \\
\hline 3 & Var3 & Renovación de tecnología & $\begin{array}{l}\text { En el año } 2015 \text { los clusters de muebles de } \\
\text { Villa El Salvador contarán con equipos de } \\
\text { vanguardia tecnológica cuya renovación } \\
\text { será periódica en plazos oportunos, } \\
\text { asimismo el control de la producción se } \\
\text { realizará mediante software diseñado para } \\
\text { el tema, la mayoría de las empresas del } \\
\text { cluster estarán conectadas en una Intranet y } \\
\text { la comunicación será fluida, así como por } \\
\text { una red externa con sus clientes. }\end{array}$ \\
\hline 4 & Var4 & $\begin{array}{l}\text { Visión del empresario con } \\
\text { respecto al mercado }\end{array}$ & $\begin{array}{l}\text { En el año } 2015 \text { la visión de mercado de los } \\
\text { empresarios de los clusters de muebles de } \\
\text { Villa El Salvador, se orientará al mercado } \\
\text { externo a la vez que se habrán posicionado } \\
\text { en el mercado nacional en ambos casos la } \\
\text { forma de venta incluirá también el comercio } \\
\text { electrónico. Asimismo el empresario } \\
\text { trabajará también con alianzas estratégicas } \\
\text { ofreciendo productos diversificados. }\end{array}$ \\
\hline 5 & Var5 & $\begin{array}{l}\text { Donaciones de } \\
\text { cooperación internacional }\end{array}$ & $\begin{array}{l}\text { En el año } 2015 \text { las donaciones de la } \\
\text { cooperación internacional hacia los clusters } \\
\text { de muebles de Villa El Salvador estarán en } \\
\text { aumento debido a los resultados positivos } \\
\text { de dichas empresas, esta se orientará tanto } \\
\text { a financiamiento de maquinaria como a } \\
\text { capacitación de operarios y plana } \\
\text { administrativa. }\end{array}$ \\
\hline
\end{tabular}

Fuente: Elaboración propia, 2005 
Cuadro 6. Probabilidades simples

\begin{tabular}{|l|r|}
\hline Hip. & Probabilités \\
\hline $4-\mathrm{H} 4$ & 0.80 \\
\hline $3-\mathrm{H} 3$ & 0.75 \\
\hline $2-\mathrm{H} 2$ & 0.70 \\
\hline $1-\mathrm{H} 1$ & 0.60 \\
\hline $5-\mathrm{H} 5$ & 0.55 \\
\hline
\end{tabular}

Fuente: Elaboración propia, 2005

Cuadro 7. Probabilidades de la «si» realización

\begin{tabular}{|c|c|c|c|c|c|}
\hline HIP. & H1 & $\mathrm{H} 2$ & $\mathrm{H} 3$ & $\mathrm{H} 4$ & H5 \\
\hline $1-\mathrm{H} 1$ & 0.6 & 0.7 & 0.76 & 0.68 & 0.55 \\
\hline $2-\mathrm{H} 2$ & 0.8 & 0.7 & 0.7 & 0.73 & 0.5 \\
\hline $3-\mathrm{H} 3$ & 0.8 & 0.65 & 0.75 & 0.6 & 0.57 \\
\hline $44-\mathrm{H} 4$ & $\overline{0.6}$ & 0.7 & 0.67 & 0.8 & 0.52 \\
\hline $5-\mathrm{H} 5$ & 0.42 & 0.38 & 0.4 & 0.48 & 0.55 \\
\hline
\end{tabular}

Fuente: Elaboración propia, 2005

Cuadro 8. Probabilidades de la «no» realización

\begin{tabular}{|c|c|c|c|c|c|}
\hline HIP. & $\mathrm{H} 1$ & $\mathrm{H} 2$ & $\mathrm{H} 3$ & $\mathrm{H} 4$ & $\mathrm{H} 5$ \\
\hline $1-\mathrm{H} 1$ & 0 & 0.62 & 0.68 & 0.46 & 0.67 \\
\hline $2-\mathrm{H} 2$ & 0 & 0 & 0.3 & 0.5 & 0.67 \\
\hline $3-\mathrm{H} 3$ & 0 & 0.25 & 0 & 0.5 & 0.7 \\
\hline$\longdiv { 4 - \mathrm { H } 4 }$ & 0.46 & $\overline{0.4}$ & 0.48 & $\overline{0}$ & 0.6 \\
\hline $5-\mathrm{H} 5$ & 0.61 & 0.67 & 0.7 & 0.5 & 0 \\
\hline
\end{tabular}

Fuente: Elaboración propia, 2005

2015 , los resultados se presentan en los cuadros 6 , 7,8 y 9 respectivamente.

Del cuadro 9 se puede deducir que los 3 primeros escenarios constituyen el núcleo tendencial (es decir donde se concentra más de la mitad de las probabilidades de realización); Por otro lado si se toma en cuenta que las hipótesis de mayor probabilidad de ocurrencia son $\mathrm{H} 1, \mathrm{H} 2, \mathrm{H} 3$ y H4 (Ver cuadro 6), se tiene los siguientes escenarios probables:

- Se cumplen todas las Hipótesis, Escenario EL PARAÍSO.

- Se cumplen $\mathrm{H} 1, \mathrm{H} 2, \mathrm{H} 3, \mathrm{H} 4$ pero no H5, Escenario EL NIRVANA.

- Se cumple sólo H5, Escenario EL HOYO.

- No se cumple ninguna, Escenario MUERTE SEGURA.

Finalmente el escenario apuesta se determina tomando como base los 4 escenarios descritos anterior- mente, a fin de tomar de algunos de ellos los elementos para formar un quinto escenario el cual es el escenario a llegar en el año 2015.

Luego de un proceso DELPHI se llega al siguiente escenario apuesta:

En el año 2015 la visión de mercado de los empresarios de los clusters de muebles de Villa EI Salvador, se orientará al mercado externo a la vez que se habrán posicionado en el mercado nacional en ambos casos la forma de venta incluirá también el comercio electrónico. Asimismo el empresario trabajará también con alianzas estratégicas ofreciendo productos diversificados.

Por otro lado el cluster de muebles de Villa El Salvador será un emporio empresarial en donde la investigación y desarrollo de nuevas tecnologías habrán alcanzado avances bastante notorios y la frecuencia 
Cuadro 9. Probabilidades de escenarios

\begin{tabular}{|l|r|r|r|}
\hline ESCENARIO & PROB & \multicolumn{1}{|l|}{$\%$} & \multicolumn{1}{l|}{ \% ACUM } \\
\hline $02-11110$ & 0.215 & 21.50 & 21.50 \\
\hline $01-11111$ & 0.195 & 19.50 & 41.00 \\
\hline $32-00000$ & 0.154 & 15.40 & 56.40 \\
\hline $29-00011$ & 0.060 & 6.00 & 62.40 \\
\hline $31-00001$ & 0.060 & 6.00 & 68.40 \\
\hline $16-10000$ & 0.036 & 3.60 & 72.00 \\
\hline $04-11100$ & 0.035 & 3.50 & 75.50 \\
\hline $15-10001$ & 0.034 & 3.40 & 78.90 \\
\hline $17-01111$ & 0.034 & 3.40 & 82.30 \\
\hline $06-11010$ & 0.032 & 3.20 & 85.50 \\
\hline $21-01011$ & 0.029 & 2.90 & 88.40 \\
\hline $11-10101$ & 0.022 & 2.20 & 90.60 \\
\hline $09-10111$ & 0.016 & 1.60 & 92.20 \\
\hline $25-00111$ & 0.016 & 1.60 & 93.80 \\
\hline $12-10100$ & 0.014 & 1.40 & 95.20 \\
\hline $30-00010$ & 0.014 & 1.40 & 96.60 \\
\hline $10-10110$ & 0.012 & 1.20 & 97.80 \\
\hline $03-11101$ & 0.010 & 1.00 & 98.80 \\
\hline $08-11000$ & 0.005 & 0.50 & 99.30 \\
\hline $22-01010$ & 0.003 & 0.30 & 99.60 \\
\hline $05-11011$ & 0.002 & 0.20 & 99.80 \\
\hline $18-01110$ & 0.002 & 0.20 & 100.00 \\
\hline $07-11001$ & 0.000 & 0.00 & 100.00 \\
\hline $13-10011$ & 0.000 & 0.00 & 100.00 \\
\hline $14-10010$ & 0.000 & 0.00 & 100.00 \\
\hline $19-01101$ & 0.000 & 0.00 & 100.00 \\
\hline $20-01100$ & 0.000 & 0.00 & 100.00 \\
\hline $23-01001$ & 0.000 & 0.00 & 100.00 \\
\hline $24-01000$ & 0.000 & 0.00 & 100.00 \\
\hline $26-00110$ & 0.000 & 0.00 & 100.00 \\
\hline $27-00101$ & 0.000 & 0.00 & 100.00 \\
\hline $28-00100$ & 0.000 & 0.00 & 100.00 \\
\hline & & & \\
\hline
\end{tabular}

Fuente: Elaboración propia, 2005

de realización es constante, contando con profesionales idóneos para el caso y con convenios con CONCYTEC, institutos y universidades nacionales $\mathrm{e}$ internacionales.

Gracias a los adelantos en investigación y la nueva visión del empresariado, el cluster contará con equipos de vanguardia tecnológica cuya renovación será periódica en plazos oportunos, asimismo el control de la producción se realizará mediante software diseñado para el tema, la mayoría de las empresas del cluster estarán conectadas en una Intranet y la comunicación será fluida, así como por una red externa con sus clientes.

Como consecuencia de lo anteriormente expuesto, los procesos de manufactura en los clusters de mue- bles de Villa El Salvador cumplirán con los estándares internacionales tanto de calidad como en buenas prácticas de manufactura, del mismo modo la producción será en serie, por otro lado la merma se verá reducida por exigentes programas de productividad otorgando mayor valor agregado al producto.

Luego un esbozo de la visión al 2015 será el siguiente:

Ser un cluster debidamente certificado, líder en exportación así como en renovación de tecnológica y de óptimos procesos de manufactura acorde a las normas internacionales, que fomente la investigación tecnológica y de nuevos productos.

\section{CONCLUSIONES}

La formulación de escenarios es un método alternativo para la generación de una Visión organizacional.

El método de la prospectiva estratégica puede ser utilizado como una forma alternativa de generar una visión de la organización.

La prospectiva estratégica genera un escenario más real en la descripción de una situación futura.

El soporte matemático del método de la prospectiva estratégica lleva a valores cuantitativos juicios cualitativos de la población consultada.

\section{RECOMENDACIONES}

A partir del escenario apuesta y tomando como base la visión formulada, redactar el Plan Estratégico Prospectivo del cluster.

Las actividades del Plan Estratégico Prospectivo deben tomar en cuenta las variables del eje estratégico determinado.

Tomar en cuenta los resultados del juego de actores a fin de analizar posibles alianzas y el resultado del comportamiento de los actores ante determinados proyectos.

\section{REFERENCIAS BIBLIOGRÁFICAS}

1. Bendahal Samuel; 2003 Multi-Issue acator análisis; Journal of Decisión Systems, Vol. 12 No. 4; Pag. 5-10.

2. Cano Alvarán Olga, 2004, Metodología para la Concertación con Actores, 1era. Edición, Escuela Superior de Administración Pública, Colombia. 
3. Godet Michel ,2000, Caja de Herramientas de la Prospectiva Estratégica, Edición Digital, LIPSOR

4. LIPSOR, 2005, MICMAC-Se poser les bonnes questions et identifier les variables clés, http:// www.3ie.org/lipsor/micmac.htm

5. LIPSOR, 2005, Analyser les stratégies d'acteurs, http://www.3ie.org/lipsor/mactor.htm

6. LIPSOR, 2005, SMIC-PROB-EXPERT, Impacts croisés probabilistes, http://www.3ie.org/lipsor/ mactor.htm

7. Mojica Francisco José, 2003, Informe del Análisis
Prospectivo del Desarrollo de la Región Bogotá Cundinamarca, Universidad Externado de Colombia, Colombia.

8. MIDEPLAN, 2003, Análisis Prospectivo de la Provincia de Valdivia para el año 2020, MIDEPLAN, Chile.

9. Ospina Toro Gildardo, 2004, Comercialización del Gas natural - Plan Estratégico Prospectivo 2004 -2015, Universidad Externado de Colombia, Colombia.

10.López Caicedo Mauricio, 2004, Estudio Prospectivo y Estratégico Municipio de Fusagasua 2020, Universidad Externado de Colombia, Colombia. 